\title{
The vigorous immune microenvironment of microsatellite instable colon cancer is balanced by multiple counter-inhibitory checkpoints
}

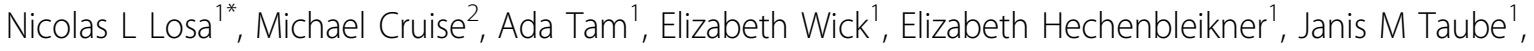 \\ Richard Blosser ${ }^{3}$, Hongni Fan ${ }^{4}$, Hao Wang ${ }^{4}$, Brandon Luber ${ }^{4}$, Ming Zhang ${ }^{4}$, Nickolas Papadopoulos ${ }^{4}$, \\ Kenneth Kinzler ${ }^{4}$, Bert Vogelstein ${ }^{4}$, Cynthia Sears ${ }^{4}$, Robert A Anders ${ }^{1}$, Drew Pardoll' ${ }^{1}$ Franck Housseau ${ }^{4}$, \\ Nicholas Siegel ${ }^{4}$
}

From 30th Annual Meeting and Associated Programs of the Society for Immunotherapy of Cancer (SITC 2015) National Harbor, MD, USA. 4-8 November 2015

We examined the immune microenvironment of primary colorectal cancer (CRC) using immunohistochemistry, laser capture microdissection/qRT-PCR, flow cytometry and functional analysis of tumor infiltrating lymphocytes. A subset of CRC displayed high infiltration with activated CD8+ CTL as well as activated Th1 cells characterized by IFN-gamma production and the Th1 transcription factor Tbet. Parallel analysis of tumor genotypes revealed that virtually all of the tumors with this active Th1/CTL microenvironment had defects in mismatch repair, as evidenced by microsatellite instability (MSI). Counterbalancing this active Th1/CTL microenvironment, MSI tumors selectively demonstrated highly up-regulated expression of multiple immune checkpoints, including five - PD-1, PD-L1, CTLA-4, LAG-3 and IDO - currently being targeted clinically with inhibitors. These findings link tumor genotype with the immune microenvironment, and explain why MSI tumors are not naturally eliminated despite a hostile Th1/CTL microenvironment. They further suggest that blockade of specific checkpoints may be selectively efficacious in the MSI subset of CRC. Our findings are the first to demonstrate a link between a genetically defined subtype of cancer and its corresponding expression of immune checkpoints in the tumor microenvironment. The mismatch repair defective subset of CRC selectively up-regulates at least 5 checkpoint molecules that are targets of inhibitors currently being clinically tested. Furthermore, our results were clinically validated in a

JJohns Hopkins University School of Medicine, Baltimore, MD, USA Full list of author information is available at the end of the article
Phase II study at Hopkins which showed mismatch-repair status as a predictor of clinical benefit to immune checkpoint blockade with pembrolizumab.

\section{Authors' details}

'Johns Hopkins University School of Medicine, Baltimore, MD, USA.

${ }^{2}$ Cleveland Clinic, Cleveland, OH, USA. ${ }^{3}$ Johns Hopkins University: School of Medicine, Baltimore, MD, USA. ${ }^{4}$ Johns Hopkins Hospital, Baltimore, MD, USA.

Published: 4 November 2015

doi:10.1186/2051-1426-3-S2-P410

Cite this article as: Losa et al.: The vigorous immune microenvironment of microsatellite instable colon cancer is balanced by multiple counterinhibitory checkpoints. Journal for Immunotherapy of Cancer 2015 3(Suppl 2):P410.

Submit your next manuscript to BioMed Central and take full advantage of:

- Convenient online submission

- Thorough peer review

- No space constraints or color figure charges

- Immediate publication on acceptance

- Inclusion in PubMed, CAS, Scopus and Google Scholar

- Research which is freely available for redistribution 